\title{
Boundedness of Singular Integral Operators with Variable Kernels on Weighted Weak Hardy Spaces
}

\author{
Hua Wang \\ College of Mathematics and Econometrics, Hunan University, Changsha 410082, China \\ Correspondence should be addressed to Hua Wang; wanghua@pku.edu.cn \\ Received 7 September 2013; Accepted 21 October 2013; Published 23 January 2014 \\ Academic Editors: W. Fei, Y. Fu, and W. Yu \\ Copyright (C) 2014 Hua Wang. This is an open access article distributed under the Creative Commons Attribution License, which \\ permits unrestricted use, distribution, and reproduction in any medium, provided the original work is properly cited. \\ Let $T_{\Omega}$ be the singular integral operator with variable kernel $\Omega(x, z)$. In this paper, by using the atomic decomposition theory of \\ weighted weak Hardy spaces, we will obtain the boundedness properties of $T_{\Omega}$ on these spaces, under some Dini type conditions \\ imposed on the variable kernel $\Omega(x, z)$.
}

\section{Introduction}

Let $S^{n-1}$ be the unit sphere in $\mathbb{R}^{n}(n \geq 2)$ equipped with the normalized Lebesgue measure $d \sigma$. A function $\Omega(x, z)$ defined on $\mathbb{R}^{n} \times \mathbb{R}^{n}$ is said to belong to $L^{\infty}\left(\mathbb{R}^{n}\right) \times L^{r}\left(S^{n-1}\right), r \geq 1$, if it satisfies the following conditions:

(A) for all $\lambda>0$ and $x, z \in \mathbb{R}^{n}, \Omega(x, \lambda z)=\Omega(x, z)$;

(B) for any $x \in \mathbb{R}^{n}, \int_{S^{n-1}} \Omega\left(x, z^{\prime}\right) d \sigma\left(z^{\prime}\right)=0$;

(C) $\|\Omega\|_{L^{\infty}\left(\mathbb{R}^{n}\right) \times L^{r}\left(S^{n-1}\right)}:=\sup _{x \in \mathbb{R}^{n}}\left(\int_{S^{n-1}}\left|\Omega\left(x, z^{\prime}\right)\right|^{r} d \sigma\left(z^{\prime}\right)\right)^{1 / r}$ $<\infty$,

where $z^{\prime}=z /|z|$ for any $z \in \mathbb{R}^{n} \backslash\{0\}$. Set $K(x, z)=$ $\Omega\left(x, z^{\prime}\right) /|z|^{n}$. In this paper, we consider the singular integral operator with variable kernel which is defined by

$$
T_{\Omega} f(x)=\text { P.V. } \int_{\mathbb{R}^{n}} K(x, x-y) f(y) d y .
$$

In $[1,2]$, Calderón and Zygmund investigated the $L^{p}$ boundedness of singular integral operators with variable kernels. They found that these operators $T_{\Omega}$ are closely related to the problem about second order elliptic partial differential equations with variable coefficients. We will denote the conjugate exponent of $p>1$ by $p^{\prime}=p /(p-1)$. In [3], Calderón and Zygmund proved the following theorem.
Theorem A (see [3]). Let $1<p, r<\infty$ satisfy

(i) $1 / r<\left(1 / p^{\prime}\right)+\left(1 / p^{\prime}(n-1)\right)$ if $1<p \leq 2$, or

(ii) $1 / r<\left(1 / p^{\prime}\right)+(1 / p(n-1))$ if $2 \leq p<\infty$.

Suppose that $\Omega(x, z) \in L^{\infty}\left(\mathbb{R}^{n}\right) \times L^{r}\left(S^{n-1}\right)$. Then there exists a constant $C>0$ independent of $f$ such that

$$
\left\|T_{\Omega}(f)\right\|_{L^{p}} \leq C\|f\|_{L^{p}} .
$$

In particular, $T_{\Omega}$ is bounded on $L^{p}\left(\mathbb{R}^{n}\right)$ for all $p \geq r^{\prime}$.

In 1971, Muckenhoupt and Wheeden [4] studied the weighted norm inequalities for $T_{\Omega}$ with power weights. In 2008, Lee et al. [5] also considered the weighted boundedness of $T_{\Omega}$ with more general weights and showed that if the kernel $K(x, y)$ satisfies the $L^{r}$-Hörmander condition with respect to $x$ and $y$ variables, respectively, then $T_{\Omega}$ is bounded on $L_{w}^{p}\left(\mathbb{R}^{n}\right)$. More precisely, they proved the following.

Theorem B (see [5]). Let $1<r<\infty$. Suppose that $\Omega(x, z) \in$ $L^{\infty}\left(\mathbb{R}^{n}\right) \times L^{r}\left(S^{n-1}\right)$ such that the following two inequalities

$$
\begin{aligned}
\sup _{\substack{x \in \mathbb{R}^{n} \\
0<|y|<R}} \sum_{k=1}^{\infty}\left(2^{k} R\right)^{n / r^{\prime}}\left(\int_{2^{k} R \leq|z|<2^{k+1} R}|K(x, z-y)-K(x, z)|^{r} d z\right)^{1 / r} \\
\quad<\infty
\end{aligned}
$$




$$
\begin{aligned}
& \sup _{\substack{x, y \in \mathbb{R}^{n} \\
0<|x-y|<R}} \sum_{k=1}^{\infty}\left(2^{k} R\right)^{n / r^{\prime}}\left(\int_{2^{k} R \leq|z|<2^{k+1} R}|K(x, z)-K(y, z)|^{r} d z\right)^{1 / r} \\
& \quad<\infty
\end{aligned}
$$

hold for all $R>0$. If $r^{\prime} \leq p<\infty$ and $w \in A_{p / r^{\prime}}$, then $T_{\Omega}$ is bounded on $L_{w}^{p}\left(\mathbb{R}^{n}\right)$.

It should be pointed out that the above $L^{r}$-Hörmander conditions on the variable kernels were also considered by Rubio de Francia et al. in [6].

In $[7,8]$, Ding et al. introduced some definitions about the variable kernel $\Omega(x, z)$ when they studied the $H^{1}-L^{1}$ boundedness of Marcinkiewicz integral. Replacing the condition (C) mentioned above, they strengthened it to the condition

$$
\left(C^{\prime}\right) \sup _{\substack{x \in \mathbb{R}^{n} \\ \rho \geq 0}}\left(\int_{S^{n-1}}\left|\Omega\left(x+\rho z^{\prime}, z^{\prime}\right)\right|^{r} d \sigma\left(z^{\prime}\right)\right)^{1 / r}<\infty .
$$

For $r \geq 1$, a function $\Omega(x, z)$ is said to satisfy the $L^{r}$-Dini condition if the conditions (A), (B), and ( $\left.\mathrm{C}^{\prime}\right)$ hold and

$$
\int_{0}^{1} \frac{\omega_{r}(\delta)}{\delta} d \delta<\infty
$$

where $\omega_{r}(\delta)$ is the integral modulus of continuity of order $r$ of $\Omega$ defined by

$$
\begin{aligned}
& \omega_{r}(\delta) \\
& : \sup _{\substack{x \in \mathbb{R}^{n} \\
\rho \geq 0}}\left(\int_{S^{n-1}} \sup _{\substack{y^{\prime} \in S^{n-1} \\
\left|y^{\prime}-z^{\prime}\right| \leq \delta}} \mid \Omega\left(x+\rho z^{\prime}, y^{\prime}\right)\right. \\
& \\
& \left.-\left.\Omega\left(x+\rho z^{\prime}, z^{\prime}\right)\right|^{r} d \sigma\left(z^{\prime}\right)\right)^{1 / r} .
\end{aligned}
$$

In order to obtain the $H_{w}^{p}-L_{w}^{p}$ boundedness of $T_{\Omega}$, Lee et al. [5] generalized the $L^{r}$-Dini condition by replacing (4) to the following stronger condition (see also [9]):

$$
\int_{0}^{1} \frac{\omega_{r}(\delta)}{\delta^{1+\alpha}} d \delta<\infty, \quad 0 \leq \alpha \leq 1 .
$$

If $\Omega$ satisfies (6) for some $r \geq 1$ and $0 \leq \alpha \leq 1$, we say that it satisfies the $L^{r, \alpha}$-Dini condition. For the special case $\alpha=0$, it reduces to the $L^{r}$-Dini condition. For $0 \leq \beta<\alpha \leq 1$, if $\Omega$ satisfies the $L^{r, \alpha}$-Dini condition, then it also satisfies the $L^{r, \beta}$. Dini condition. We thus denote by $\operatorname{Din}_{\alpha}^{r}\left(S^{n-1}\right)$ the class of all functions which satisfy the $L^{r, \beta}$-Dini condition for all $0<\beta<$ $\alpha$.

Theorem C (see [5]). Let $0<\alpha \leq 1$ and $n /(n+\alpha)<p<1$. Suppose $\Omega \in \operatorname{Din}_{\alpha}^{r}\left(S^{n-1}\right)$ such that (3) hold for a certain large number $r>1$. If $w^{r^{\prime}} \in A_{(p+(p \alpha / n)-(1 / r)) r^{\prime}}$, then there exists a constant $C>0$ independent of $f$ such that

$$
\left\|T_{\Omega}(f)\right\|_{L_{w}^{p}} \leq C\|f\|_{H_{w}^{p}} .
$$

It is easy to check that

$$
\operatorname{Din}_{\alpha}^{r}\left(S^{n-1}\right) \subset \operatorname{Din}_{\alpha}^{s}\left(S^{n-1}\right), \quad \text { if } 1 \leq s<r<\infty .
$$

Then for $0<\alpha \leq 1$, we define

$$
\operatorname{Din}_{\alpha}^{\infty}\left(S^{n-1}\right)=\bigcap_{r \geq 1} \operatorname{Din}_{\alpha}^{r}\left(S^{n-1}\right) .
$$

The main purpose of this paper is to study the corresponding estimates of $T_{\Omega}$ on the weighted weak Hardy spaces $W H_{w}^{p}\left(\mathbb{R}^{n}\right)$ (see Section 2 for its definition). We now present our main result as follows.

Theorem 1. Let $0<\alpha \leq 1, n /(n+\alpha)<p \leq 1$, and $w \in$ $A_{p(1+(\alpha / n))}$. Suppose $\Omega \in \operatorname{Din}_{\alpha}^{\infty}\left(S^{n-1}\right)$ such that (3) hold. Then there exists a constant $C>0$ independent of $f$ such that

$$
\left\|T_{\Omega}(f)\right\|_{W L_{w}^{p}} \leq C\|f\|_{W H_{w}^{p}} .
$$

\section{Notations and Preliminaries}

The definition of $A_{p}$ class was first used by Muckenhoupt [10], Hunt et al. [11], and Coifman and Fefferman [12] in the study of weighted $L^{p}$ boundedness of Hardy-Littlewood maximal functions and singular integrals. Let $w$ be a nonnegative, locally integrable function defined on $\mathbb{R}^{n}$; all cubes are assumed to have their sides parallel to the coordinate axes. We say that $w \in A_{p}, 1<p<\infty$, if

$$
\begin{array}{r}
\left(\frac{1}{|Q|} \int_{Q} w(x) d x\right)\left(\frac{1}{|Q|} \int_{Q} w(x)^{-1 /(p-1)} d x\right)^{p-1} \leq C, \\
\text { for every cube } Q \subseteq \mathbb{R}^{n},
\end{array}
$$

where $C$ is a positive constant which is independent of the choice of $Q$. For the case $p=1, w \in A_{1}$, if

$$
\begin{array}{r}
\frac{1}{|Q|} \int_{Q} w(x) d x \leq C \cdot \operatorname{ess} \inf _{x \in Q} w(x), \\
\text { for every cube } Q \subseteq \mathbb{R}^{n} .
\end{array}
$$

The smallest value of $C>0$ such that the above inequalities hold is called the $A_{p}$ characteristic constant of $w$ and denoted by $[w]_{A_{p}}$. For the case $p=\infty, w \in A_{\infty}$, if it satisfies the $A_{p}$ condition for some $1<p<\infty$.

A weight function $w$ is said to belong to the reverse Hölder class $R H_{s}$ if there exist two constants $s>1$ and $C>0$ such that the following reverse Hölder inequality holds:

$$
\left(\frac{1}{|Q|} \int_{Q} w(x)^{s} d x\right)^{1 / s} \leq C\left(\frac{1}{|Q|} \int_{Q} w(x) d x\right),
$$


It is well known that if $w \in A_{p}$ with $1<p<\infty$, then $w \in$ $A_{r}$ for all $r>p$ and $w \in A_{q}$ for some $1<q<p$. We thus write $q_{w} \equiv \inf \left\{q>1: w \in A_{q}\right\}$ to denote the critical index of $w$. Moreover, if $w \in A_{p}$ with $1 \leq p<\infty$, then there exists $s>1$ such that $w \in R H_{s}$. It follows directly from Hölder's inequality that $w \in R H_{r}$ implies $w \in R H_{s}$ for all $1<s<r$.

Given a cube $Q$ and $\lambda>0, \lambda Q$ stands for the cube with the same center as $Q$ whose side length is $\lambda$ times that of $Q . Q=$ $Q\left(x_{0}, r\right)$ denotes the cube centered at $x_{0}$ with side length $r$. For a weight function $w$ and a measurable set $E$, we denote the Lebesgue measure of $E$ by $|E|$ and set the weighted measure $w(E)=\int_{E} w(x) d x$.

We give the following results that will be used in the sequel.

Lemma 2 (see [13]). Let $w \in A_{q}$ with $q \geq 1$. Then, for any cube $Q$, there exists an absolute constant $C>0$ such that

$$
w(2 Q) \leq C w(Q) .
$$

In general, for any $\lambda>1$, one has

$$
w(\lambda Q) \leq C \cdot \lambda^{n q} w(Q),
$$

where $C$ does not depend on $Q$ or $\lambda$.

Given a weight function $w$ on $\mathbb{R}^{n}$, for $0<p<\infty$, we denote by $L_{w}^{p}\left(\mathbb{R}^{n}\right)$ the weighted space of all functions $f$ satisfying

$$
\|f\|_{L_{w}^{p}}=\left(\int_{\mathbb{R}^{n}}|f(x)|^{p} w(x) d x\right)^{1 / p}<\infty .
$$

When $p=\infty, L_{w}^{\infty}\left(\mathbb{R}^{n}\right)$ will be taken to mean $L^{\infty}\left(\mathbb{R}^{n}\right)$ and

$$
\|f\|_{L_{w}^{\infty}}=\|f\|_{L^{\infty}}=\underset{x \in \mathbb{R}^{n}}{\operatorname{ess} \sup }|f(x)| .
$$

We also denote by $W L_{w}^{p}\left(\mathbb{R}^{n}\right)$ the weighted weak $L^{p}$ space which is formed by all measurable functions $f$ satisfying

$$
\|f\|_{W L_{w}^{p}}=\sup _{\lambda>0} \lambda \cdot w\left(\left\{x \in \mathbb{R}^{n}:|f(x)|>\lambda\right\}\right)^{1 / p}<\infty .
$$

Let us now turn to the weighted weak Hardy spaces. The (unweighted) weak $H^{p}$ spaces have first appeared in the work of Fefferman et al. [14], which are the intermediate spaces between two Hardy spaces through the real method of interpolation. The atomic decomposition characterization of weak $H^{1}$ space on $\mathbb{R}^{n}$ was given by Fefferman and Soria in [15]. Later, Liu [16] established the weak $H^{p}$ spaces on homogeneous groups for the whole range $0<p \leq 1$. The corresponding results related to $\mathbb{R}^{n}$ can be found in [17]. For the boundedness properties of some operators on weak Hardy spaces, we refer the readers to [18-24]. In 2000, Quek and Yang [25] introduced the weighted weak Hardy spaces $W H_{w}^{p}\left(\mathbb{R}^{n}\right)$ and established their atomic decompositions. Moreover, by using the atomic decomposition theory of $W H_{w}^{p}\left(\mathbb{R}^{n}\right)$, Quek and Yang [25] also obtained the boundedness of Calderón-Zygmund type operators on these weighted spaces.

We write $\mathcal{S}\left(\mathbb{R}^{n}\right)$ to denote the Schwartz space of all rapidly decreasing infinitely differentiable functions and
$S^{\prime}\left(\mathbb{R}^{n}\right)$ to denote the space of all tempered distributions, that is, the topological dual of $\mathcal{S}\left(\mathbb{R}^{n}\right)$. Let $w \in A_{\infty}, 0<p \leq 1$ and $N=\left[n\left(q_{w} / p-1\right)\right]$. Define

$$
\begin{aligned}
& \mathscr{A}_{N, w}=\left\{\varphi \in \mathcal{S}\left(\mathbb{R}^{n}\right):\right. \\
&\left.\sup _{x \in \mathbb{R}^{n}|\alpha| \leq N+1} \sup _{1}(1+|x|)^{N+n+1}\left|D^{\alpha} \varphi(x)\right| \leq 1\right\},
\end{aligned}
$$

where $\alpha=\left(\alpha_{1}, \ldots, \alpha_{n}\right) \in(\mathbb{N} \cup\{0\})^{n},|\alpha|=\alpha_{1}+\cdots+\alpha_{n}$ and

$$
D^{\alpha} \varphi=\frac{\partial^{|\alpha|} \varphi}{\partial x_{1}^{\alpha_{1}} \cdots \partial x_{n}^{\alpha_{n}}} .
$$

For any given $f \in \mathcal{S}^{\prime}\left(\mathbb{R}^{n}\right)$, the grand maximal function of $f$ is defined by

$$
G_{w} f(x)=\sup _{\varphi \in \mathscr{A}_{N, w}|y-x|<t} \sup _{\mid}\left|\left(\varphi_{t} * f\right)(y)\right| .
$$

Then we can define the weighted weak Hardy space $W H_{w}^{p}\left(\mathbb{R}^{n}\right)$ by $W H_{w}^{p}\left(\mathbb{R}^{n}\right)=\left\{f \in \mathcal{S}^{\prime}\left(\mathbb{R}^{n}\right): G_{w} f \in W L_{w}^{p}\left(\mathbb{R}^{n}\right)\right\}$. Moreover, we set $\|f\|_{W H_{w}^{p}}=\left\|G_{w} f\right\|_{W L_{w}^{p}}$.

Theorem 3 (see [25]). Let $0<p \leq 1$ and $w \in A_{\infty}$. For every $f \in W H_{w}^{p}\left(\mathbb{R}^{n}\right)$, there exists a sequence of bounded measurable functions $\left\{f_{k}\right\}_{k=-\infty}^{\infty}$ such that

(i) $f=\sum_{k=-\infty}^{\infty} f_{k}$ in the sense of distributions;

(ii) each $f_{k}$ can be further decomposed into $f_{k}=\sum_{i} b_{i}^{k}$, where $\left\{b_{i}^{k}\right\}$ satisfies that

(a) each $b_{i}^{k}$ is supported in a cube $Q_{i}^{k}$ with $\sum_{i} w\left(Q_{i}^{k}\right) \leq c 2^{-k p}$ and $\sum_{i} \chi_{Q_{i}^{k}}(x) \leq c$. Here $\chi_{E}$ denotes the characteristic function of the set $E$ and $c \sim\|f\|_{W H_{w}^{p}}^{p} ;$

(b) $\left\|b_{i}^{k}\right\|_{L^{\infty}} \leq C 2^{k}$, where $C>0$ is independent of $i$ and $k$;

(c) $\int_{\mathbb{R}^{n}} b_{i}^{k}(x) x^{\alpha} d x=0$ for every multi-index $\alpha$ with $|\alpha| \leq\left[n\left(q_{w} / p-1\right)\right]$.

Conversely, if $f \in \mathcal{S}^{\prime}\left(\mathbb{R}^{n}\right)$ has a decomposition satisfying (i) and (ii), then $f \in W H_{w}^{p}\left(\mathbb{R}^{n}\right)$. Moreover, one has $\|f\|_{W H_{w}^{p}}^{p} \sim$ c.

Throughout this paper $C$ always denotes a positive constant, which is independent of the main parameters and not necessarily the same at each occurrence.

\section{Proof of Theorem 1}

Following the same arguments as in the proof of Lemma 5 in [26], we can also establish the following lemma on the variable kernel $\Omega(x, z)$ (see $[5,8]$ ).

Lemma 4. Let $r \geq 1$. Suppose that $\Omega(x, z) \in L^{\infty}\left(\mathbb{R}^{n}\right) \times$ $L^{r}\left(S^{n-1}\right)$ satisfies the $L^{r}$-Dini condition in Section 1. If there 
exists a constant $0<\gamma \leq 1 / 2$ such that $|y|<\gamma R$, then for any $x_{0} \in \mathbb{R}^{n}$, one has

$$
\begin{gathered}
\left(\int_{R \leq|x|<2 R}\left|K\left(x+x_{0}, x-y\right)-K\left(x+x_{0}, x\right)\right|^{r} d x\right)^{1 / r} \\
\leq C \cdot R^{-n / r^{\prime}}\left(\frac{|y|}{R}+\int_{|y| / 2 R}^{|y| / R} \frac{\omega_{r}(\delta)}{\delta} d \delta\right)
\end{gathered}
$$

where the constant $C>0$ is independent of $R$ and $y$.

We are now in a position to give the proof of Theorem 1 .

Proof of Theorem 1. For any given $\lambda>0$, we may choose $k_{0} \in$ $\mathbb{Z}$ such that $2^{k_{0}} \leq \lambda<2^{k_{0}+1}$. For every $f \in W H_{w}^{p}\left(\mathbb{R}^{n}\right)$, then by Theorem 3 , we can write

$$
f=\sum_{k=-\infty}^{\infty} f_{k}=\sum_{k=-\infty}^{k_{0}} f_{k}+\sum_{k=k_{0}+1}^{\infty} f_{k}:=F_{1}+F_{2},
$$

where $F_{1}=\sum_{k=-\infty}^{k_{0}} f_{k}=\sum_{k=-\infty}^{k_{0}} \sum_{i} b_{i}^{k}, F_{2}=\sum_{k=k_{0}+1}^{\infty} f_{k}=$ $\sum_{k=k_{0}+1}^{\infty} \sum_{i} b_{i}^{k}$, and $\left\{b_{i}^{k}\right\}$ satisfies (a)-(c) in Theorem 3 . Then we have

$$
\begin{aligned}
\lambda^{p} \cdot w & \left(\left\{x \in \mathbb{R}^{n}:\left|T_{\Omega}(f)(x)\right|>\lambda\right\}\right) \\
\leq & \lambda^{p} \cdot w\left(\left\{x \in \mathbb{R}^{n}:\left|T_{\Omega}\left(F_{1}\right)(x)\right|>\frac{\lambda}{2}\right\}\right) \\
& +\lambda^{p} \cdot w\left(\left\{x \in \mathbb{R}^{n}:\left|T_{\Omega}\left(F_{2}\right)(x)\right|>\frac{\lambda}{2}\right\}\right) \\
= & I_{1}+I_{2} .
\end{aligned}
$$

First we claim that the following inequality holds:

$$
\left\|F_{1}\right\|_{L_{w}^{2}} \leq C \cdot \lambda^{1-p / 2}\|f\|_{W H_{w}^{p}}^{p / 2}
$$

In fact, since supp $b_{i}^{k} \subseteq Q_{i}^{k}=Q\left(x_{i}^{k}, r_{i}^{k}\right)$ and $\left\|b_{i}^{k}\right\|_{L^{\infty}} \leq C 2^{k}$ according to Theorem 3 , then it follows directly from Minkowski's inequality that

$$
\begin{aligned}
\left\|F_{1}\right\|_{L_{w}^{2}} & \leq \sum_{k=-\infty}^{k_{0}} \sum_{i}\left\|b_{i}^{k}\right\|_{L_{w}^{2}} \\
& \leq \sum_{k=-\infty}^{k_{0}} \sum_{i}\left\|b_{i}^{k}\right\|_{L^{\infty}} w\left(Q_{i}^{k}\right)^{1 / 2} .
\end{aligned}
$$

For each $k \in \mathbb{Z}$, by using the bounded overlapping property of the cubes $\left\{Q_{i}^{k}\right\}$ and the fact that $1-p / 2>0$, we thus obtain

$$
\begin{aligned}
\left\|F_{1}\right\|_{L_{w}^{2}} & \leq C \sum_{k=-\infty}^{k_{0}} 2^{k}\left(\sum_{i} w\left(Q_{i}^{k}\right)\right)^{1 / 2} \\
& \leq C \sum_{k=-\infty}^{k_{0}} 2^{k(1-p / 2)}\|f\|_{W H_{w}^{p}}^{p / 2} \\
& \leq C \sum_{k=-\infty}^{k_{0}} 2^{\left(k-k_{0}\right)(1-p / 2)} \cdot \lambda^{1-(p / 2)}\|f\|_{W H_{w}^{p}}^{p / 2} \\
& \leq C \cdot \lambda^{1-p / 2}\|f\|_{W H_{w}^{p / 2}}^{p}
\end{aligned}
$$

Since $w \in A_{p(1+(\alpha / n))}$ and $p(1+(\alpha / n)) \leq 1+(\alpha / n)<2$, then we have $w \in A_{2}$. In this case, we know that there exists a number $s>1$ such that $w \in R H_{s}$. More specifically, by using the sharp reverse Hölder's inequality for $A_{2}$ weights obtained recently in [27], we find that for $w \in A_{2}$,

$$
w \in R H_{s} \quad \text { with } s=1+\frac{1}{2^{n+5}[w]_{A_{2}}} .
$$

Observe that $\Omega \in \operatorname{Din}_{\alpha}^{\infty}\left(S^{n-1}\right)$, then we are able to find a positive number $r>1$ large enough such that $r>$ $\max \left\{s^{\prime},(2 n) /(n-\alpha)\right\}$ and $\Omega \in \operatorname{Din}_{\alpha}^{r}\left(S^{n-1}\right)$. By the choice of $r$, we can easily check that $2 / r^{\prime}>1+\alpha / n \geq p(1+\alpha / n)$, which implies $w \in A_{2 / r^{\prime}}$. Hence, by using Theorem $\mathrm{B}$, we know that $T_{\Omega}$ is bounded on $L_{w}^{2}\left(\mathbb{R}^{n}\right)$. This fact together with Chebyshev's inequality and (25) yields

$$
\begin{aligned}
I_{1} & \leq \lambda^{p} \cdot \frac{4}{\lambda^{2}}\left\|T_{\Omega}\left(F_{1}\right)\right\|_{L_{w}^{2}}^{2} \\
& \leq C \cdot \lambda^{p-2}\left\|F_{1}\right\|_{L_{w}^{2}}^{2} \\
& \leq C\|f\|_{W H_{w}^{p}}^{p} .
\end{aligned}
$$

We now turn our attention to the estimate of $I_{2}$. Setting

$$
A_{k_{0}}=\bigcup_{k=k_{0}+1}^{\infty} \bigcup_{i} \widetilde{Q_{i}^{k}}
$$

where $\widetilde{Q_{i}^{k}}=Q\left(x_{i}^{k}, \tau^{\left(k-k_{0}\right) /(n+\alpha)}(2 \sqrt{n}) r_{i}^{k}\right)$ and $\tau$ is a fixed positive number such that $1<\tau<2$. Thus, we can further decompose $I_{2}$ as

$$
\begin{aligned}
I_{2} \leq & \lambda^{p} \cdot w\left(\left\{x \in A_{k_{0}}:\left|T_{\Omega}\left(F_{2}\right)(x)\right|>\frac{\lambda}{2}\right\}\right) \\
& +\lambda^{p} \cdot w\left(\left\{x \in\left(A_{k_{0}}\right)^{c}:\left|T_{\Omega}\left(F_{2}\right)(x)\right|>\frac{\lambda}{2}\right\}\right) \\
= & I_{2}^{\prime}+I_{2}^{\prime \prime} .
\end{aligned}
$$

Let us first deal with the term $I_{2}^{\prime}$. Since $w \in A_{p(1+(\alpha / n))}$, then by Lemma 2 , we can deduce that

$$
\begin{aligned}
I_{2}^{\prime} & \leq \lambda^{p} \sum_{k=k_{0}+1}^{\infty} \sum_{i} w\left(\widetilde{Q_{i}^{k}}\right) \\
& \leq C \cdot \lambda^{p} \sum_{k=k_{0}+1}^{\infty} \tau^{\left(k-k_{0}\right) p} \sum_{i} w\left(Q_{i}^{k}\right) \\
& \leq C\|f\|_{W H_{w}^{p}}^{p} \sum_{k=k_{0}+1}^{\infty}\left(\frac{\tau}{2}\right)^{\left(k-k_{0}\right) p} \\
& \leq C\|f\|_{W H_{w}^{p}}^{p}
\end{aligned}
$$


On the other hand, it follows immediately from Chebyshev's inequality that

$$
\begin{aligned}
I_{2}^{\prime \prime} & \leq 2^{p} \int_{\left(A_{k_{0}}\right)^{c}}\left|T_{\Omega}\left(F_{2}\right)(x)\right|^{p} w(x) d x \\
& \leq 2^{p} \sum_{k=k_{0}+1}^{\infty} \sum_{i} \int_{\left(\widetilde{Q}_{i}^{k}\right)^{c}}\left|T_{\Omega}\left(b_{i}^{k}\right)(x)\right|^{p} w(x) d x \\
& =2^{p} \sum_{k=k_{0}+1}^{\infty} \sum_{i} J_{i}^{k} .
\end{aligned}
$$

Now denote $\tau_{i, \ell}^{k}=2^{\ell-1} \tau^{\left(k-k_{0}\right) /(n+\alpha)} \sqrt{n} r_{i}^{k}, \widetilde{Q_{i, \ell}^{k}}=Q\left(x_{i}^{k}, \tau_{i, \ell}^{k}\right)$, and

$$
E_{i, \ell}^{k}=\left\{x \in \mathbb{R}^{n}: \tau_{i, \ell}^{k} \leq\left|x-x_{i}^{k}\right|<2 \tau_{i, \ell}^{k}\right\}, \quad \ell=1,2, \ldots
$$

An application of Hölder's inequality gives us that

$$
\begin{aligned}
J_{i}^{k} & \leq \sum_{\ell=1}^{\infty} \int_{E_{i, \ell}^{k}}\left|T_{\Omega}\left(b_{i}^{k}\right)(x)\right|^{p} w(x) d x \\
& \leq \sum_{\ell=1}^{\infty}\left(\int_{E_{i, \ell}^{k}} w(x) d x\right)^{1-p}\left(\int_{E_{i, \ell}^{k}}\left|T_{\Omega}\left(b_{i}^{k}\right)(x)\right| w(x) d x\right)^{p} .
\end{aligned}
$$

Let $q=p(1+(\alpha / n))$ for simplicity. Then for any $n /(n+\alpha)<$ $p \leq 1$ and $w \in A_{q}$ with $q>1$, we can easily see that $\left[n\left(q_{w} / p-\right.\right.$ $1)]=0$. Hence, by the cancellation condition of $b_{i}^{k} \in L^{\infty}\left(\mathbb{R}^{n}\right)$, we get

$$
\begin{aligned}
& \int_{E_{i, \ell}^{k}}\left|T_{\Omega}\left(b_{i}^{k}\right)(x)\right| w(x) d x \\
& =\int_{E_{i, \ell}^{k}}\left|\int_{Q_{i}^{k}}\left[K(x, x-y)-K\left(x, x-x_{i}^{k}\right)\right] b_{i}^{k}(y) d y\right| w(x) d x \\
& \leq \int_{Q_{i}^{k}}\left\{\int_{E_{i, \ell}^{k}}\left|K(x, x-y)-K\left(x, x-x_{i}^{k}\right)\right| w(x) d x\right\}\left|b_{i}^{k}(y)\right| d y \\
& \leq\left\|b_{i}^{k}\right\|_{L^{\infty}}\left|Q_{i}^{k}\right|\left(\int_{E_{i, \ell}^{k}}\left|K(x, x-y)-K\left(x, x-x_{i}^{k}\right)\right| w(x) d x\right) .
\end{aligned}
$$

When $y \in Q_{i}^{k}$ and $x \in\left(\widetilde{Q_{i}^{k}}\right)^{c}$, then a trivial computation shows that

$$
\left|x-x_{i}^{k}\right| \geq \tau^{\left(k-k_{0}\right) /(n+\alpha)} \sqrt{n} r_{i}^{k}>\sqrt{n} r_{i}^{k} \geq 2\left|y-x_{i}^{k}\right|
$$

We also observe that $w \in R H_{s}$ and $r>s^{\prime}$, then $w \in R H_{r^{\prime}}$. Using Hölder's inequality, the estimate (37), and Lemma 4, we can see that for any $y \in Q_{i}^{k}$, the integral of the above expression is dominated by

$$
\begin{aligned}
& \left(\int_{E_{i, \ell}^{k}}\left|K(x, x-y)-K\left(x, x-x_{i}^{k}\right)\right|^{r} d x\right)^{1 / r} \\
& \times\left(\int_{E_{i, \ell}^{k}} w(x)^{r^{\prime}} d x\right)^{1 / r^{\prime}} \\
& \leq C \cdot \frac{w\left(\widetilde{Q_{i, \ell+1}^{k}}\right)}{\left|\widetilde{Q_{i, \ell+1}^{k}}\right|^{1 / r}}\left(\int_{\tau_{i, \ell}^{k} \leq|x|<2 \tau_{i, \ell}^{k}} \mid K\left(x+x_{i}^{k}, x-\left(y-x_{i}^{k}\right)\right)\right. \\
& \left.-\left.K\left(x+x_{i}^{k}, x\right)\right|^{r} d x\right)^{1 / r} \\
& \leq C \cdot \frac{w\left(\widetilde{Q_{i, \ell+1}^{k}}\right)}{\left|\widetilde{Q_{i, \ell+1}^{k}}\right|^{1 / r}} \cdot\left(\tau_{i, \ell}^{k}\right)^{-n / r^{\prime}} \\
& \times\left(\frac{\left|y-x_{i}^{k}\right|}{\tau_{i, \ell}^{k}}+\int_{\left|y-x_{i}^{k}\right| / 2 \tau_{i, \ell}^{k}}^{\left|y-x_{i}^{k}\right| / \tau_{i, \ell}^{k}} \frac{\omega_{r}(\delta)}{\delta} d \delta\right) \\
& \leq C \cdot \frac{w\left(\widetilde{Q_{i, \ell+1}^{k}}\right)}{\left|\widetilde{Q_{i, \ell+1}^{k}}\right|^{1 / r}} \cdot\left(\tau_{i, \ell}^{k}\right)^{-n / r^{\prime}} \\
& \times\left(\frac{\left|y-x_{i}^{k}\right|}{\tau_{i, \ell}^{k}}+\frac{\left|y-x_{i}^{k}\right|^{\alpha}}{\left(\tau_{i, \ell}^{k}\right)^{\alpha}} \times \int_{\left|y-x_{i}^{k}\right| / 2 \tau_{i, \ell}^{k}}^{\left|y-x_{i}^{k}\right| / \tau_{i, \ell}^{k}} \frac{\omega_{r}(\delta)}{\delta^{1+\alpha}} d \delta\right) \\
& \leq C \cdot \frac{w\left(\widetilde{Q_{i, \ell+1}^{k}}\right)}{\left|\widetilde{Q_{i, \ell+1}^{k}}\right|} \\
& \cdot\left(\frac{1}{2^{\ell} \tau^{\left(k-k_{0}\right) /(n+\alpha)}}+\left[\frac{1}{2^{\ell} \tau^{\left(k-k_{0}\right) /(n+\alpha)}}\right]^{\alpha} \int_{0}^{1} \frac{\omega_{r}(\delta)}{\delta^{1+\alpha}} d \delta\right) \\
& \leq C \cdot\left(1+\int_{0}^{1} \frac{\omega_{r}(\delta)}{\delta^{1+\alpha}} d \delta\right) \cdot \frac{w\left(\widetilde{Q_{i, \ell+1}^{k}}\right)}{\left|\widetilde{Q_{i, \ell+1}^{k}}\right|}\left(\frac{1}{2^{\ell} \tau^{\left(k-k_{0}\right) /(n+\alpha)}}\right)^{\alpha} .
\end{aligned}
$$

Recall that $\left\|b_{i}^{k}\right\|_{L^{\infty}} \leq C 2^{k}$. From the above estimate (38), it follows that

$$
\begin{aligned}
J_{i}^{k} \leq C \cdot 2^{k p} \sum_{\ell=1}^{\infty} w & \left(\widetilde{Q_{i, \ell+1}^{k}}\right)\left(\frac{\left|Q_{i}^{k}\right|}{\left|\widetilde{Q_{i, \ell+1}^{k}}\right|}\right)^{p} \\
& \times\left(\frac{1}{2^{\ell} \tau^{\left(k-k_{0}\right) /(n+\alpha)}}\right)^{\alpha p} .
\end{aligned}
$$


In addition, for $w \in A_{q}$ with $q>1$, then we can take a sufficiently small number $\varepsilon>0$ such that $w \in A_{q-\varepsilon}$. Thus, by using Lemma 2 again, we finally obtain

$$
\begin{aligned}
& J_{i}^{k} \leq C \cdot 2^{k p} w\left(Q_{i}^{k}\right) \\
& \times \sum_{\ell=1}^{\infty}\left(2^{\ell} \tau^{\left(k-k_{0}\right) /(n+\alpha)} \sqrt{n}\right)^{n(q-\varepsilon)-n p} \\
& \times\left(\frac{1}{2^{\ell} \tau^{\left(k-k_{0}\right) /(n+\alpha)}}\right)^{\alpha p} \\
& \leq C \cdot 2^{k p} w\left(Q_{i}^{k}\right) \sum_{\ell=1}^{\infty}\left(2^{\ell} \tau^{\left(k-k_{0}\right) /(n+\alpha)}\right)^{-n \varepsilon} \\
& \leq C \cdot 2^{k p} w\left(Q_{i}^{k}\right)\left(\tau^{\left(k-k_{0}\right) /(n+\alpha)}\right)^{-n \varepsilon} .
\end{aligned}
$$

Therefore

$$
\begin{aligned}
I_{2}^{\prime \prime} & \leq C \sum_{k=k_{0}+1}^{\infty} \sum_{i} 2^{k p}\left(\tau^{\left(k-k_{0}\right) /(n+\alpha)}\right)^{-n \varepsilon} w\left(Q_{i}^{k}\right) \\
& \leq C\|f\|_{W H_{w}^{p}}^{p} \sum_{k=k_{0}+1}^{\infty}\left(\tau^{\left(k-k_{0}\right) /(n+\alpha)}\right)^{-n \varepsilon} \\
& \leq C\|f\|_{W H_{w}^{p}}^{p}
\end{aligned}
$$

Combining the above inequality (41) with (29) and (32), and then taking the supremum over all $\lambda>0$, we conclude the proof of Theorem 1 .

\section{Conflict of Interests}

The author declares that there is no conflict of interests regarding the publication of this paper.

\section{Acknowledgment}

The author would like to thank Professor J. Duoandikoetxea for pointing out the reference [6].

\section{References}

[1] A. P. Calderón and A. Zygmund, "On a problem of Mihlin," Transactions of the American Mathematical Society, vol. 78, pp. 209-224, 1955.

[2] A. P. Calderón and A. Zygmund, "On singular integrals," American Journal of Mathematics, vol. 78, pp. 289-309, 1956.

[3] A. P. Calderón and A. Zygmund, "On singular integrals with variable kernels," Applicable Analysis, vol. 7, no. 3, pp. 221-238, 1978.

[4] B. Muckenhoupt and R. L. Wheeden, "Weighted norm inequalities for singular and fractional integrals," Transactions of the American Mathematical Society, vol. 161, pp. 249-258, 1971.

[5] M. Y. Lee, C. C. Lin, Y. C. Lin, and D. Y. Yan, "Boundedness of singular integral operators with variable kernels," Journal of Mathematical Analysis and Applications, vol. 348, no. 2, pp. 787796, 2008.

[6] J. L. Rubio de Francia, F. J. Ruiz, and J. L. Torrea, "CalderónZygmund theory for operator-valued kernels," Advances in Mathematics, vol. 62, no. 1, pp. 7-48, 1986.
[7] Y. Ding, C. C. Lin, and Y. C. Lin, "Erratum: "On Marcinkiewicz integral with variable kernels”, Vol. 53 (2004), 805-822," Indiana University Mathematics Journal, vol. 56, no. 2, pp. 991-994, 2007.

[8] Y. Ding, C. C. Lin, and S. L. Shao, "On the Marcinkiewicz integral with variable kernels," Indiana University Mathematics Journal, vol. 53, no. 3, pp. 805-821, 2004.

[9] C. C. Lin, Y. C. Lin, X. Tao, and X. Yu, "The boundedness of Marcinkiewicz integral with variable kernel," Illinois Journal of Mathematics, vol. 53, no. 1, pp. 197-217, 2009.

[10] B. Muckenhoupt, "Weighted norm inequalities for the Hardy maximal function," Transactions of the American Mathematical Society, vol. 165, pp. 207-226, 1972.

[11] R. A. Hunt, B. Muckenhoupt, and R. L. Wheeden, "Weighted norm inequalities for the conjugate function and Hilbert transform," Transactions of the American Mathematical Society, vol. 176, pp. 227-251, 1973.

[12] R. R. Coifman and C. Fefferman, "Weighted norm inequalities for maximal functions and singular integrals," Studia Mathematica, vol. 51, pp. 241-250, 1974.

[13] J. García-Cuerva and J. L. Rubio de Francia, Weighted Norm Inequalities and Related Topics, North-Holland Publishing, Amsterdam, The Netherlands, 1985.

[14] C. Fefferman, N. M. Rivière, and Y. Sagher, "Interpolation between $H^{p}$ spaces: the real method," Transactions of the American Mathematical Society, vol. 191, pp. 75-81, 1974.

[15] R. Fefferman and F. Soria, "The space weak $H^{1}$," Studia Mathematica, vol. 85, no. 1, p. 1-16 (1987), 1986.

[16] H. P. Liu, "The weak $H^{p}$ spaces on homogeneous groups," in Harmonic Analysis, vol. 1494 of Lecture Notes in Mathematics, pp. 113-118, Springer, 1991.

[17] S. Z. Lu, Four Lectures on Real $H^{p}$ Spaces, World Scientific, River Edge, NJ, USA, 1995.

[18] Y. Ding, S. Z. Lu, and S. L. Shao, "Integral operators with variable kernels on weak Hardy spaces," Journal of Mathematical Analysis and Applications, vol. 317, no. 1, pp. 127-135, 2006.

[19] Y. Ding, S. Z. Lu, and Q. Y. Xue, "Marcinkiewicz integral on Hardy spaces," Integral Equations and Operator Theory, vol. 42, no. 2, pp. 174-182, 2002.

[20] Y. Ding, S. Z. Lu, and Q. Y. Xue, "Parametrized Littlewood-Paley operators on Hardy and weak Hardy spaces," Mathematische Nachrichten, vol. 280, no. 4, pp. 351-363, 2007.

[21] Y. Ding, S. Z. Lu, and Q. Y. Xue, "Parametrized area integrals on Hardy spaces and weak Hardy spaces," Acta Mathematica Sinica, vol. 23, no. 9, pp. 1537-1552, 2007.

[22] Y. Ding and X. F. Wu, "Weak Hardy space and endpoint estimates for singular integrals on space of homogeneous type," Turkish Journal of Mathematics, vol. 34, no. 2, pp. 235-247, 2010.

[23] H. P. Liu, "The wavelet characterization of the space weak $H^{1}$," Studia Mathematica, vol. 103, no. 1, pp. 109-117, 1992.

[24] X. X. Tao, X. Yu, and S. Y. Zhang, "Marcinkiewicz integrals with variable kernels on Hardy and weak Hardy spaces," Journal of Function Spaces and Applications, vol. 8, no. 1, pp. 1-16, 2010.

[25] T. S. Quek and D. C. Yang, "Calderón-Zygmund-type operators on weighted weak Hardy spaces over $\mathbb{R}^{n}$," Acta Mathematica Sinica, vol. 16, no. 1, pp. 141-160, 2000.

[26] D. S. Kurtz and R. L. Wheeden, "Results on weighted norm inequalities for multipliers," Transactions of the American Mathematical Society, vol. 255, pp. 343-362, 1979.

[27] D. Chung, M. C. Pereyra, and C. Perez, "Sharp bounds for general commutators on weighted Lebesgue spaces," Transactions of the American Mathematical Society, vol. 364, no. 3, pp. 11631177, 2012. 


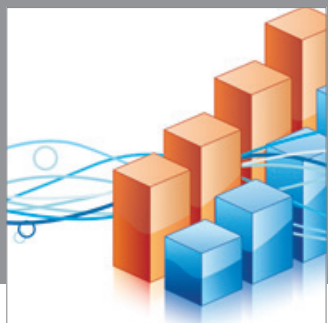

Advances in

Operations Research

mansans

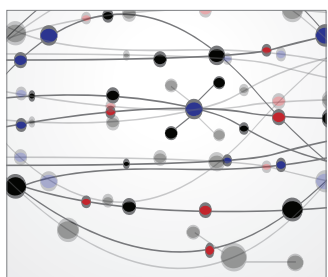

The Scientific World Journal
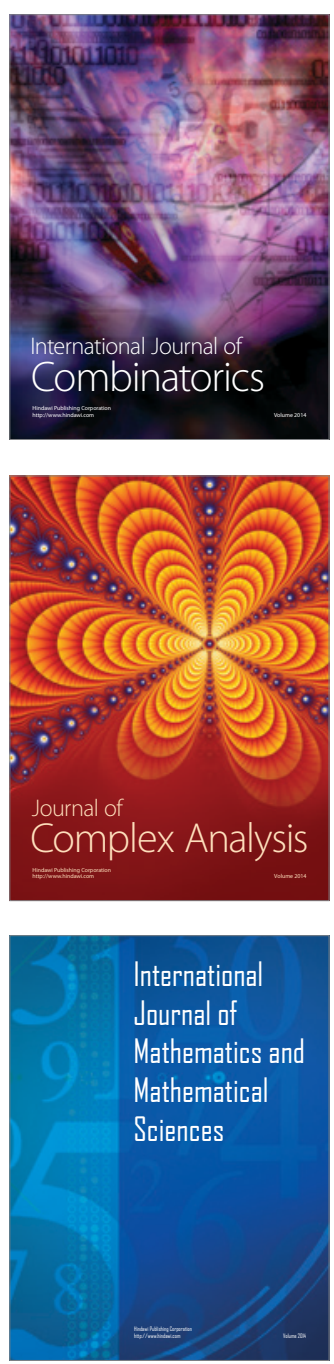
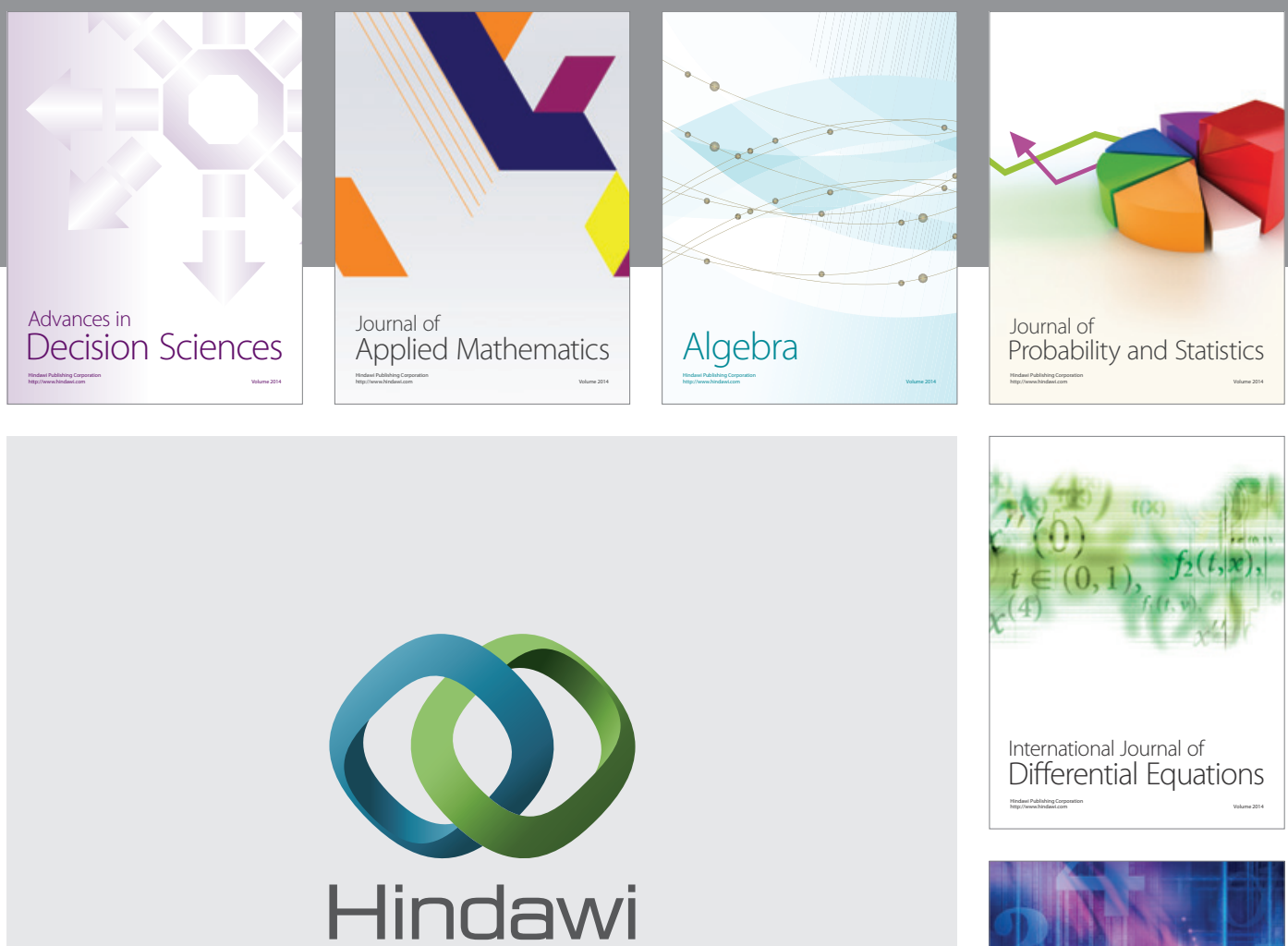

Submit your manuscripts at http://www.hindawi.com
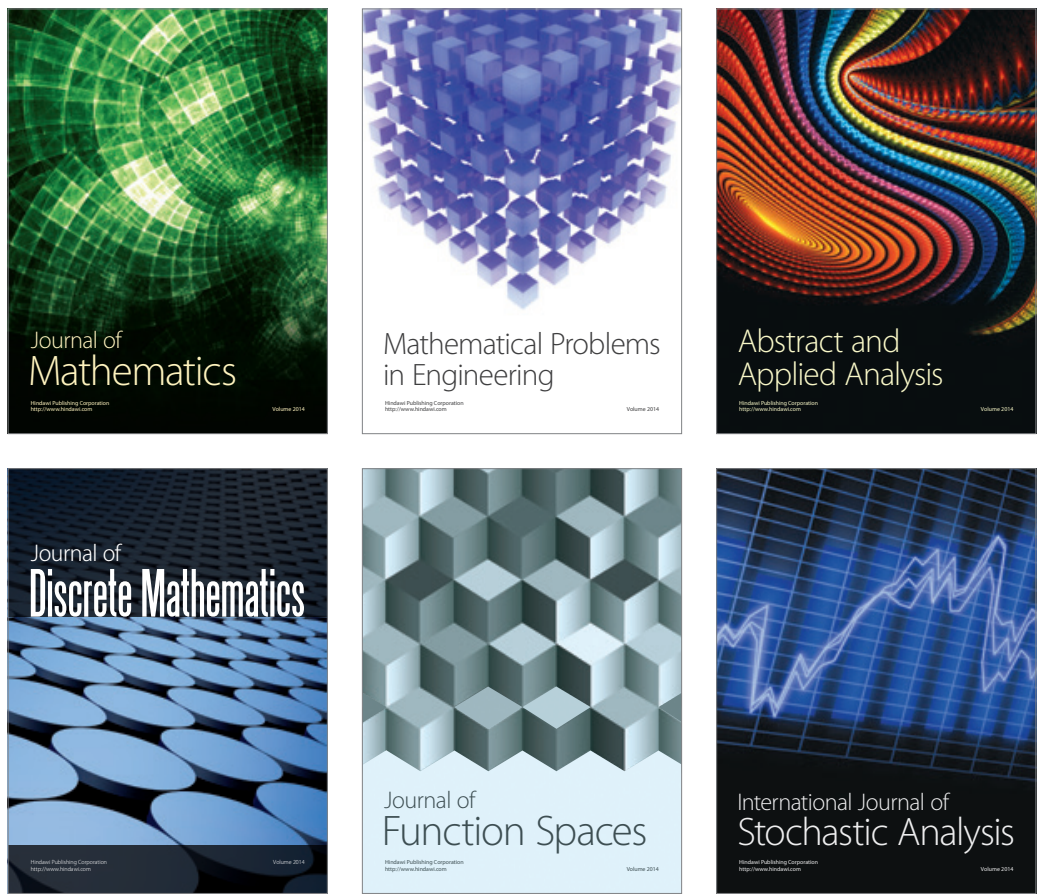

Journal of

Function Spaces

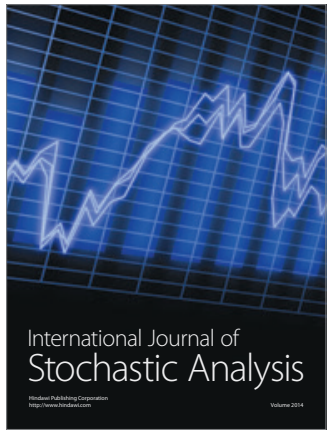

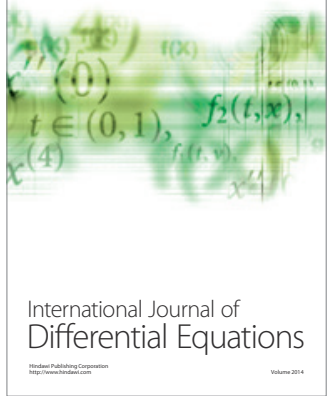
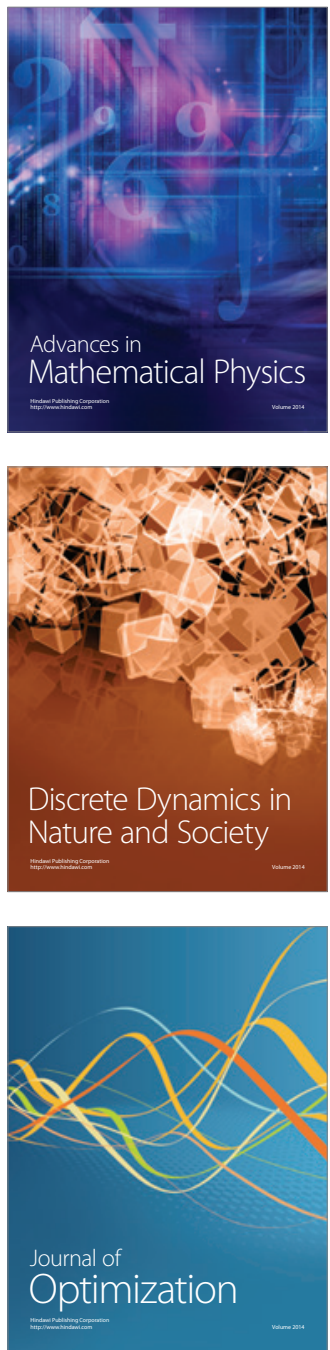